\title{
DRIVING CYCLE ESTIMATION AND VALDATION FOR LUDHIANA CITY, INDIA
}

\author{
Poojari Yugendar ${ }^{1}$, Kalaga Ramachandra $\mathbf{R a o}^{2}$, Geetam Tiwari ${ }^{3}$ \\ ${ }^{1}$ Department of Civil Engineering, Chaitanya Bharathi Institute of Technology, Hyderabad-500075, \\ Telangana State, India \\ ${ }^{2,3}$ Department of Civil Engineering, Indian Institute of Technology, Delhi, Hauz Khas, New Delhi, \\ Delhi-110016, India
}

Received 30 January 2020; accepted 29 March 2020

\begin{abstract}
Traffic behavior of a city or any area can represent using driving cycle. In India, driving cycles were developed to test Indian vehicle emission standards but not considering higher speed and acceleration of vehicles. The assumption was all vehicle activities to be similar and considered traffic as homogeneous. But in India, traffic was heterogeneous each vehicle activity is different. In this study, the driving cycle was developed for estimating vehicular emissions and fuel consumption. Driving cycle is developed using five parameters namely percentage of acceleration, deceleration, idle, cruise and average speed of vehicles. Micro-trips were used to develop a driving cycle and these micro-trips are extracted from real-world data. K-means clustering method was used to cluster the micro-trips. The microtrips which are nearest to the cluster centre represented as representative micro trips. These representative micro-trips are used for the development of the driving cycle. The driving cycles were developed and compared route and mode wise. The developed driving cycle was compared with Delhi driving cycle. It was observed that acceleration, deceleration rates were high compared to Delhi driving cycle. This methodology can be useful for heterogeneous traffic condition. The developed driving cycles can be easily identified the driving characteristics and vehicle emissions when testing on chassis dynamometers.
\end{abstract}

Keywords: fuel consumption, emission, micro trips, cruise, idle.

\section{Introduction}

The main sources of air pollution are vehicular emissions, industrial emissions, dust and storm, emissions from air craft and jets. Vehicular emissions are the main sources of atmospheric pollution in cities. Many cities are facing problems like traffic congestion, vehicular emission because of increase in vehicular population. According to national environmental engineering research institute, $60-70 \%$ of air pollution is caused because of vehicular emissions in all major cities. The factors mainly affecting the vehicular emissions are road characteristics and vehicular characteristics. Vehicular emissions are depending mainly on type of engine, age of vehicle, type of fuel and driving cycle. Automotive Research Association of India (ARAI) developed Indian driving cycle (IDC) based on assumption that traffic is homogeneous but actually which is heterogeneous. In IDC, average speed and acceleration considered as $42 \mathrm{~km} / \mathrm{h}$

${ }^{1}$ Corresponding author: poojariyugendar_civil@cbit.ac.in 
and $0.65 \mathrm{~m} / \mathrm{s}^{2}$, but in many cities, the speed inside city is limited greater than $42 \mathrm{~km} / \mathrm{h}$. Generally, the driving pattern is varies from city to city and region to region. The driving cycles developed in certain region may not be suitable to other regions. Generally, driving cycle was defined as "speed-time profile for a vehicle driving under a specified condition for a given city or region". Fuel consumption and vehicular emissions are measured using driving cycles for a particular area.

Driving cycles were divided into two types: transient and polygonal driving cycles. Transient driving cycles were developed based on the on road driving data (Melbourne peak cycle and US FTP cycles). Transient driving cycles were developed to urban or rural areas based on specific driving characteristics. These characteristics differ from one area to another and also differ in same city. The polygonal driving cycles were developed by compose of a sequence of steady state modes like constant acceleration and speed driving modes (Japanese and ECE cycles). These cycles do not explain the real driving behavior due to the effect of vehicle modal operations.

According to their uses, driving cycles were classified into two types: legislative driving cycles and non legislative cycles (Tong et al., 1999). In legislative driving cycles, the driving conditions within their respective boundaries were considered and these cycles were delivered to government for vehicle emission controls. Non legislative cycles were developed for the estimation of fuel consumption and vehicle emissions.

According to the qualitative descriptions driving cycles were divided into three categories: urban driving cycles, sub urban/ composite driving cycles and highway driving cycle. Urban driving cycles have lower average speed and higher acceleration rates. It was observed that, regular stop and go operations in urban areas with heavy traffic flows and many intersections. Composite driving cycles have slightly higher average speed than urban driving cycles but smaller acceleration rates. Highway driving cycles have higher average speed but smaller acceleration rates. It shows that, very less traffic flows and limited stops and minimum number of intersections.

In this study, transient driving cycle was used. This study explains the development of transient driving cycle using five parameters like percentage of acceleration, deceleration, idle, cruise and average speed of vehicles for heterogeneous traffic condition.

\section{Literature Review}

Kent et al. (1978) developed a driving cycle for Sydney to estimate exhaust emissions. Average speed, root mean square acceleration and percentage idle time were used for driving cycle development. Hung et al. (2005) developed a model to analyze the driving characteristics by using three step approach. In the first step, 10 driving parameters were selected and used in the model. In second step, speed acceleration probability matrix (SAPM) was derived to know the distributions of speed and acceleration. In the third step, driving cycles were developed using 10 driving parameters and SAPM. Hung et al. (2007) presented a methodology to develop a driving cycle to estimate the vehicle emissions. Speed data was collected using on board and chase car technique. For the construction of driving cycle, a lot of parameters were selected. The 
developed driving cycle estimation model was validated using performance value and speed acceleration probability distribution (SAPD).

Nutramon and Supachart (2009) and Tamsanya et al. (2009) developed a new method for construction of a driving cycle for Bangkok. Data was collected on few routes and these routes selected that the traffic on these routes represent the traffic conditions on entire Bangkok traffic. Various driving parameters were used in driving cycle construction and these parameters derived from actual traffic data. The driving cycle was constructed by connecting series of several real world micro trips obtained from real traffic data. Kamble et al. (2009) develop an urban driving cycle for estimating vehicular emissions and fuel consumption. Five parameters were considered to develop a driving cycle namely percentage of acceleration, deceleration, idle, cruise and average speed. The driving cycle was constructed by connecting series of several real world micro trips obtained from real traffic data. Saleh et al. (2009) developed an urban and rural driving cycle for Hong Kong for cars. 12 parameters were selected for constructing driving cycle for cars. The representative driving cycle was selected by calculating the absolute sums of the relative error $\left(S_{j}\right)$. The driving cycle with minimum relative error is the representative driving cycle. Saleh et al. (2010) developed a driving cycle for Edinburgh and Delhi using same methodology.

Tong and Hung (2010) proposed a framework on development of driving cycle based on 101 transient driving cycles. In 101 driving cycles, Asian driving was the slowest one and European driving is the fastest and smoothest. Traffic activity patterns should be considered to determining the test routes. Speed data collection methods namely onboard measurement, chase car techniques or their hybrid were explained. Most of the researchers ignored the tendency of zero change in acceleration while constructing driving cycle. Application of succession probability was explained at second-bysecond level.

In this study, driving cycle was developed for heterogeneous traffic condition using five parameters. The next sections describe the methodology and in the subsequent section generation of micro-trips were discussed.

\section{Methodology}

A methodology was proposed for developing a real world driving cycle using micro trips and these micro trips represents the existing traffic conditions. The parameters were used to develop driving cycles are percentage of acceleration, percentage of deceleration, percentage of idle, percentage cruise and average speed. The methodology consists of driving data collection, micro-trips generation, data analysis and driving cycle construction.

\subsection{Driving Data}

Ludhiana city, in India was selected for this study. Ludhiana city is an important urban center in Punjab and a rapidly growing metropolis of the country. The selected routes and distances are tabulated in Table 1. On-board measurement technique was used to collect the speed-time data. This technique was used for large scale studies and data was collected directly and more accurately. Trimble GPS instrument was used for data collection. The instrument having high sensitivity GPS receiver with 
a field computer powered by the windows mobile version 6.1 operating system. The final data collection schedule was tabulated in Table 2. There are 2 out of 7 days as weekends that gives the almost $28 \%$ of the total week. So this supports way of dividing it into three trips from weekends and 6 trips from weekdays.

Table 1

Selected Routes Represent Real Traffic Conditions

\begin{tabular}{|l|l|l|l|}
\hline S. No & From & To & Distance $(\mathbf{k m})$ \\
\hline 1 & Jalandhar bypass & Dhandhari & 10.3 \\
\hline 2 & Samarla chowk & Bhai bala chowk & 8.0 \\
\hline 3 & Bhai bala chowk & Thakkarwal & 6.4 \\
\hline 4 & Gill chowk & Gill bypass road & 4.5 \\
\hline Total & & & 31 \\
\hline
\end{tabular}

Table 2

Data Collection Schedule

\begin{tabular}{|c|c|c|c|c|}
\hline \multirow{2}{*}{ Day type } & \multicolumn{3}{|c|}{ Number of trips } & \multirow{2}{*}{ Total } \\
\cline { 2 - 5 } & Morning peak & Afternoon peak & Evening peak & 6 \\
\hline Weekdays & 2 & 2 & 2 & 3 \\
\hline Weekends & 1 & 1 & 1 & 9 \\
\hline Total & 3 & 3 & 3 & 6 \\
\hline
\end{tabular}

\subsection{Generation of Micro-Trips}

The speed-time profile is divided into smaller units called as micro-trips for driving cycle development. Micro trip is defined as "trip between two consecutive time points at which vehicle is stopped". In previous studies, micro-trips were developed by manual count. In this study, micro trips were developed using MATLAB. Each micro-trip includes five parameters like percentage acceleration, percentage deceleration, and percentage cruise, percentage idle and average speed which were used for driving cycle development.

\subsection{Data Analysis}

Data analysis was done in two parts. In the first part, base data was analyzed and micro-trips were constructed. Base data analysis involves the development of speed-acceleration frequency matrix and normalized speed-acceleration matrix. The frequency of occurrence of acceleration, deceleration, cruise and idle corresponding to a speed values represented in a matrix form is called speed-acceleration frequency matrix. Normalized speed acceleration matrix was obtained by dividing each cell by total time and divide by 100 . Micro-trips were constructed using computer program. In the second part, micro-trips were clustered using K-means clustering algorithm.

The speed-acceleration frequency matrix (SAFD) of three- wheeler on GT road was shown in Fig. 1. The normalized speedacceleration matrix from the base data (SAPD) was shown in Fig. 2. The sample micro trip for three-wheeler was shown in Fig. 3. 

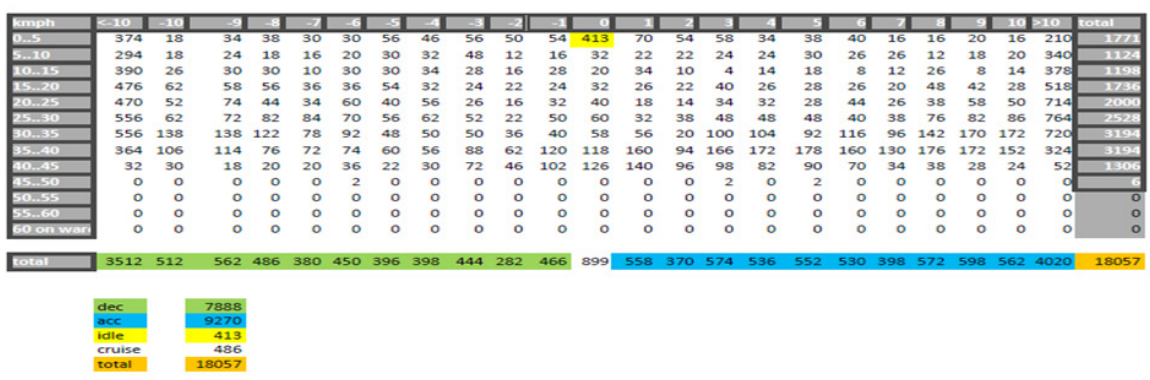

Fig. 1.

The Speed-Acceleration Frequency Matrix (SAFD) from Base Data for 3W (Sample)
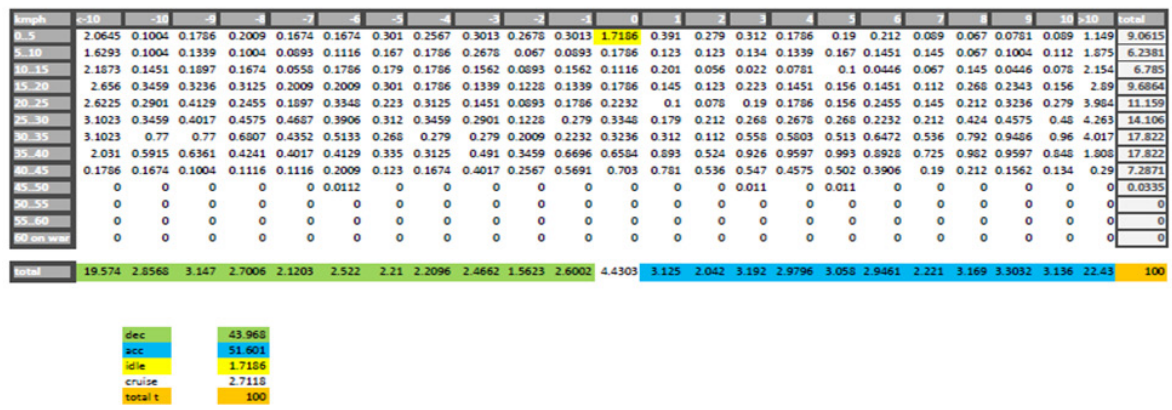

Fig. 2.

The Normalized Speed-Acceleration Matrix (SAPD) from the Base Data for $3 W$ (Sample)

Micro trip (23)

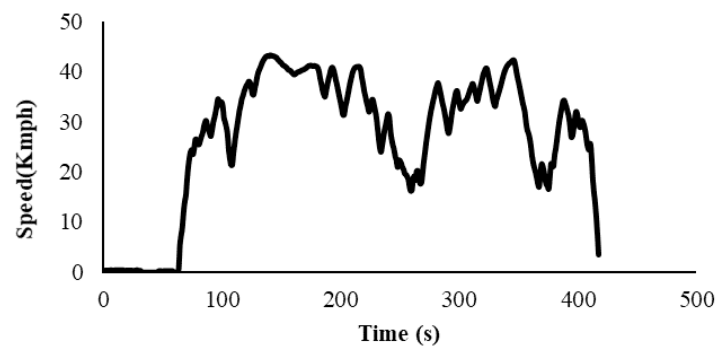

Fig. 3.

Micro Trip (23) for Three-Wheeler

\subsection{Construction of Driving Cycle}

Representative micro-trips were determined for each cluster for driving cycle development.
The micro-trips nearer to cluster centers were selected as representative micro-trips and this process was to select enough micro-trips from all clusters to develop candidate driving cycle. 
Performance value (PV) and sum squared difference (SSD) were used to select best driving cycle from all the candidate driving cycles. Performance value is the absolute sum of the difference between candidate cycle and target value. If the PV value is less, the real world driving pattern was represented more accurately. The candidate cycle with smallest PV value will be selected as the most representative driving cycle for the corresponding group. The PV value is given in Eq (1):

$$
\mathrm{PV}=\left|\theta_{i}-\theta_{T}\right| . \mathrm{W}_{\mathrm{T}}
$$

Where, $\mathrm{W}_{\mathrm{T}}$ is the transpose of the row weight vector corresponding to the set of statistics. $\theta_{\mathrm{i}}$ and $\theta_{\mathrm{T}}$ represents the five target parameters corresponding to the base data and candidate driving cycle. The weights assigned to five parameters were considered to be equal weight age because of the unavailability of the weights.

Mathematically SSD is given in Eq. (2). The candidate driving cycle with smallest SSD value is selected as representative driving cycle for the corresponding group.

$\mathrm{SSD}=\sum_{i=1}^{N_{S}} \sum_{j=1}^{N_{a}}\left(p_{i j}-q_{i j}\right)^{2}$
Where $\mathrm{N}_{\mathrm{s}}$ is the speed classes, $\mathrm{N}_{\mathrm{a}}$ is the number of acceleration classes, $\mathrm{p}_{\mathrm{ij}}$ is the $\mathrm{ij}^{\text {th }}$ entry of the candidate cycle, and $\mathrm{q}_{\mathrm{ij}}$ is the $\mathrm{ij}^{\text {th }}$ entry of SAPD of the overall driving speed profiles. It is the sum of the squared differences of the corresponding cells of the base SAPD and candidate driving cycles SAPD.

\section{Evaluation of the Driving Cycle}

The developed driving cycle was compared with existing driving cycle and tabulated in Table 3. The comparison was done based on key parameters like percentage of acceleration, percentage of deceleration, percentage of idle, percentage of cruise and average speed. It was observed that, the percentage acceleration and percentage deceleration were high for developed driving cycle compared to other driving cycles. It means that, vehicle release more emissions and more pollution occurred if accelerations and decelerations were more. The average speed was observed to be more compared to others except Japanese and US driving cycles. The percentage of cruise and idle were observed to be less compared to others.

Table 3

Comparison of Driving Cycle Parameters from Ludhiana to other Studies

\begin{tabular}{|l|l|l|l|l|l|}
\hline Driving Cycle Type & Speed & Cruise & Idle & Acceleration & Deceleration \\
\hline ECE & 18.35 & 29.23 & 30.7 & 21.54 & 18.46 \\
\hline Japanese & 25.61 & 20.88 & 31.65 & 26.15 & 21.32 \\
\hline US 75 & 34.1 & 20.4 & 18 & 33.1 & 28.5 \\
\hline BDC & 17.7 & 23.8 & 37.7 & 15.3 & 23.2 \\
\hline IDC & 21.9 & 10.43 & 16.52 & 38.89 & 34.26 \\
\hline Pune & 19.55 & 56.25 & 18.09 & 14.18 & 11.48 \\
\hline Present Study & 24.83 & 2.392 & 5.404 & 50.997 & 41.174 \\
\hline
\end{tabular}




\section{Conclusions}

Driving cycle was developed for measuring the fuel emissions and consumptions. The driving cycle's were constructed mode wise and route wise and compared. The parameters like the percentage acceleration, percentage deceleration, idle, cruise and the average speed were used for developing the driving cycle. Driving cycle was constructed based on micro-trips and these micro-trips were obtained by dividing the base data. $\mathrm{K}$-means clustering technique was used to group the micro-trips based on similarity. SAPD and SAPD matrixes were constructed and these were used for dividing the whole data into speed-acceleration ranges form. Based on PV and SSD representative driving cycle was selected from all candidate driving cycles. For all modes, (car, three-wheeler, and two-wheeler) time spent in acceleration and deceleration in Gill road is less compared to other driving cycles. For all routes, GT road, $\mathrm{NH}$ 95, Pakhwoal road, and Gill road time spent in acceleration and deceleration were high for two wheelers compared to other driving cycles. The developed driving cycle was compared with other driving cycles. Time spent in acceleration and deceleration modes for Ludhiana driving cycles are found to be significantly higher than Delhi driving cycles and also all other driving cycles.

\section{References}

Hung, W.T.; Tam, K.M.; Lee, C.P.; Chan, L.Y.; Cheung, C.S. 2005. Comparison of driving characteristics in cities of Pearl River Delta, China Journal of atmospheric Environment 39(4): 615-625.
Hung, W.T.; Tong, H.Y.; Lee, C.P.; Ha, K.; Pao, L.Y. 2007. Development of a practical driving cycle construction methodology: a case study in Hong Kong, Journal of Transportation Research Part D 12(2): 115-128.

Kamble, S.H.; Mathew, T.V.; Sharma, G.K. 2009. Development of real-world driving cycle: case study of Pune, India, Journal of Transportation Research PartD 14(2): 132140.

Kent, J.H.; Allen, G.H.; Rule, G. 1978. A driving cycle for Sydney, Transportation Research 12(3): 147-152.

Nutramon, T.; Chungpaibulpatana, S. 2009. Influence of driving cycles on exhaust emissions and fuel consumption of gasoline passenger car in Bangkok, Journal of Environmental Sciences 21(5): 604-611.

Saleh, W.; Kumar, R.; Kirby, H.; Kumar, P. 2009. Real world driving cycle for motorcycles in Edinburgh, Journal of Transportation Research Part D 14(5): 326-333.

Saleh, W.; Kumar, R.; Sharma, A. 2010. Real-World Driving Cycle for Motorcycles: A Comparative Study between Delhi and Edinburgh, World Journal of Science, Technology \& Sustainable Development 7(3): 263-274.

Tamsanya, S.; Chunpaibulpatana, S.; Limmeechokchai, B. 2009. Development of a driving cycle for the measurement of fuel consumption and exhaust emissions of automobiles in Bangkok during peak periods, International Journal of Automotive Technology 10(2):251-264.

Tong, H.Y.; Hung, W.T.; Cheung, C.S. 1999. Development of a driving cycle for Hong Kong, Journal of Atmospheric Environment 33(15): 2323-2335.

Tong, H.Y.; Hung, W.T. 2010. Framework for Developing Driving Cycles with On Road Driving Data, Transport Reviews 30(5): 589-615. 